\title{
Ablactación temprana como factor de riesgo para neumonía muy grave
}

\author{
Early ablation as a risk factor for very severe pneumonia
}

\author{
Oscar Valdelamar Espitia, MD ${ }^{1}$ Eliana Paola Valderrama Martínez, MD² \\ Andrea Carolina Zárate Vergara, MD ${ }^{3}$ IRINa Suley TiRado PÉRez, MD ${ }^{4}$
}

\section{Resumen}

INTRODUCCIÓN: la neumonía es una entidad frecuente en pediatría y sigue siendo una de las principales causas de morbimortalidad. En su estudio, se han encontrado factores de riesgo, como la ablactación temprana, que es uno de los más importantes.

MATERIAL Y MÉTODOS: estudio descriptico y retrospectivo. Se tomaron los datos con base en las historias clínicas de niños menores de 5 años. Los datos fueron almacenados y estudiados en el programa Excel, y mostrados en tablas y porcentajes.

RESULTADOS: el estudio tuvo una muestra de 93 historias. Los resultados fueron distribuidos de la siguiente manera: el porcentaje de aquellos que eran menores de 2 meses y presentaron neumonía muy grave fue menor que en el grupo de aquellos mayores de 2 meses a los 5 años: $4,30 \%$, que corresponden a 4 pacientes, y $95,7 \%$, que corresponden a 89 pacientes. En el grupo de pacientes menores de 2 meses con neumonía muy grave que tuvieron o no ablactación temprana es igual. Debido a la escasa población incluida en esta categoría, no es posible determinar una relación de la ablactación temprana como factor de riesgo para la presentación de neumonía muy grave. Según los resultados obtenidos en el estudio, se puede aprecia que, en el grupo de mayores de 2 meses a 5 años, el porcentaje de pacientes enfermos con antecedentes de ablactación temprana fue mayor $(58,4 \%)$ versus un porcentaje menor $(41,5 \%)$ de los pacientes que no tenían este antecedente.

DISCUSIÓN: evidenciar que más del 50\% de la población del estudio presentó neumonía y ablactación temprana indica una relación importante entre esta y la presentación de neumonía muy grave en niños, sobre todo en los mayores de 2 meses hasta los 5 años. Se puede concluir que hay una relación entre ablactación temprana y el padecimiento de una infección respiratoria grave, por lo que la lactancia materna sigue siendo un factor protector contra varias patologías, entre estas, las de afectación respiratoria.

Palabras claves: pediatría, neumonía, lactancia materna.

\section{Abstract}

INTRODUCTION TO: Pneumonia is a frequent cause in pediatrics that continues to be one of the main causes of morbidity and mortality; in its study, risk factors have been found, so ablation remains a problem.

MATERIAL AND METHODS: Descriptive, retrospective study, data considered in the database in clinical records in children under the 5 years, stored data and studies in Excel program shown in tables and percentages.

\footnotetext{
${ }^{1}$ Médico Pediatra, Universidad de Cartagena. Hospital Infantil "Napoleón Franco Pareja" (HINFP).

${ }^{2}$ Médico Interno, Corporación Universitaria Rafael "Núñez". Hospital Infantil "Napoleón Franco Pareja" (HINFP).

3 Médico Epidemiólogo. Residente de Cuidado Intensivo Pediátrico, Universidad de Santander.

4 Médico Epidemiólogo. Magíster en Cuidado Paliativo. Residente, Cuidado Intensivo Pediátrico, Universidad de Santander.

Autor de correspondencia

Irina Suley Tirado Pérez. El Bosque, Sector F, apartamento 302, torre 9, Floridablanca, Santander. Tel.: +57 (314)559.86.97. Correo electrónico: iri29@hotmail.com

Recibido: 01/12/17. Aceptado: 20/12/17.
} 
RESULTS: The study tube a sample of 93 stories, whose result were distributed in the following manner the percentage of those who were under the age of 2 months and had very severe pneumonia been lower than in the group of older than 2 months to 5 years: $4.30 \%$, corresponding to 4 patients and $95.7 \%$ corresponding to 89 patients, respectively. Group of patients younger than 2 months with very severe pneumonia that had or not early ablactacion is the same, due to the scarce population included in this category, it is not possible to determine a relationship of the early ablactacion as risk factor for the presentation of very severe pneumonia. According to the results of the study can be seen that the percentage of patients with a history of early ablactacion was higher in the Group of older than two months to 5 years $(58.4 \%)$ versus one smaller percentage $(41.5 \%)$ of the patients who did not have this antecedent.

DISCUSSION: To show that over $50 \%$ of the study population presenting pneumonia and early ablactacion indicates a significant relationship between this and the presentation of very severe pneumonia in children, mostly over the age of 2 months to 5 years, we can conclude one relationship between early ablactacion and suffering from a respiratory infection severe, by which breastfeeding continues being a protective factor against several diseases including respiratory involvement.

Keywords: pediatric, pneumonia, breast feeding.

\section{Introducción}

La neumonía es un proceso inflamatorio del parénquima pulmonar, generalmente de tipo infeccioso. Muchos factores se relacionan con la presentación de neumonía muy grave; entre ellos, la ablactación temprana tiene un aporte importante. Se consideró relevante realizar este proyecto en el Hospital Infantil "Napoleón Franco Pareja" (HINFP) de alta complejidad, en la ciudad de Cartagena, Colombia, teniendo presente que la principal causa de consulta en pediatría es por infecciones respiratorias, entre ellas la neumonía.

La neumonía adquirida en la comunidad es una enfermedad común potencialmente fatal. La principal causa de muerte en niños de ambos sexos menores de 5 años en los países subdesarrollados y en vía de desarrollo, como el nuestro, es la neumonía muy grave. La lactancia materna exclusiva hasta los 6 meses disminuye el riesgo de este tipo de neumonía. Actualmente, el promedio de lactancia materna exclusiva para Colombia es de solo 1,8 meses. Este país tiene como compromiso del milenio disminuir la mortalidad infantil en dos tercios.

El desarrollo de este proyecto contribuye a sensibilizar a los trabajadores de la salud, pediatras, residentes, internos, estudiantes, personal de enfermería y otros, sobre la importancia de educar y estimular a las madres lactantes a practicar la lactancia materna exclusiva durante los primeros 6 meses del nacimiento del niño, contribuyendo así a disminuir la incidencia de neumonía muy grave $(1,2)$.

\section{Materiales y métodos}

El presente es un estudio retrospectivo de corte transversal realizado en el Hospital Infantil "Napoleón Franco Pareja" durante el período comprendido entre 1 de julio y 31 de diciembre de 2016. Para propósitos de este análisis, la población estudiada se agrupó en dos categorías en función de la edad: menores de 2 meses y mayores de 2 meses hasta 5 años.

La recolección de la información se hizo a partir de historias clínicas, y fue responsabilidad del grupo de investigación del proyecto, a través del uso de encuestas prediseñadas. En $90 \%$ se obtuvo los datos a partir de la información aportada por las historias clínicas y el $10 \%$ por llamadas telefónicas a los acudientes. Los datos obtenidos se agruparon y tabularon mediante el programa Excel, con elaboración de tablas y porcentajes a partir de los resultados.

Para escoger la muestra, que en este caso fue por conveniencia, de las 800 historias clínicas de los pacientes con diagnóstico de neumonía se identificaron las características clínicas en los niños menores de 2 meses y mayores de 2 meses hasta 5 años; luego se clasificaron según los criterios de neumonía muy grave de la Atención Integrada de las Enfermedades Prevalentes de la Infancia (AIEPI), obteniéndose así un total de 93 historias clínicas para realizar el estudio. Después se correlacionó este diagnóstico con el antecedente de ablactación temprana, definida como la suspensión de la lactancia materna exclusiva antes de los 6 meses re- 
comendados por la Organización Mundial de la Salud (OMS). Durante la realización del estudio se tuvieron en cuenta los criterios éticos, en los cuales se garantizó la confidencialidad y que la información obtenida solo se utilizara para fines académicos. No se hicieron juicios de valor. Para la obtención de la muestra se tuvieron en cuenta los siguientes criterios:

\section{Criterios de inclusión}

- Paciente con diagnóstico de neumonía muy grave: definido como la presentación de insuficiencia respiratoria aguda (IRA), más cualquiera de los signos y síntomas consignados en las encuestas

- Pacientes ingresados dentro del período comprendido entre 1 de julio y 31 de diciembre de 2016

- Pacientes con edades comprendidas entre 0 meses y 5 años

- Pacientes con historias clínicas bien diligenciadas (completas).

\section{Criterios de exclusión}

- Pacientes que no cumplieron con los criterios de neumonía muy grave establecidos por AIEPI

- Pacientes ingresados fuera del período de estudio

- Pacientes con edades mayores de 5 años

- Pacientes a los cuales no se les pudo confirmar el tiempo de lactancia materna exclusiva, ya sea porque no se encontraba consignado en la historia clínica o por la imposibilidad de comunicación con el acudiente.

\section{Resultados}

Según los resultados descritos en la Tabla 1, el porcentaje de pacientes menores de 2 meses con neumonía muy grave que tuvo o no ablactación temprana es igual. Debido a la escasa población incluida en esta categoría no es posible determinar una relación de la ablactación temprana como factor de riesgo para la presentación de neumonía muy grave en este grupo de edad.
Tabla 1. Resultados en los pacientes menores de 2 meses

\begin{tabular}{|cccc|}
\hline Sin ablactación temprana & \multicolumn{2}{c|}{$\begin{array}{c}\text { Con ablactación } \\
\text { temprana }\end{array}$} \\
$\begin{array}{c}\text { Total de } \\
\text { historias } \\
\text { clínicas }\end{array}$ & Porcentaje & $\begin{array}{c}\text { Total de } \\
\text { historias } \\
\text { clínicas }\end{array}$ & Porcentaje \\
\hline 2 & $2,15 \%$ & 2 & $2,15 \%$ \\
\hline
\end{tabular}

El porcentaje de pacientes mayores de 2 meses hasta los 5 años con neumonía muy grave que tuvo ablactación temprana es mayor que los que no tuvieron: $58,4 \%$ vs. 41,5\%, respectivamente (Tabla 2).

Tabla 2. Resultado en los pacientes mayores de 2 meses hasta los 5 años

\begin{tabular}{|cccc|}
\hline Sin ablactación temprana & \multicolumn{2}{c|}{$\begin{array}{c}\text { Con ablactación } \\
\text { temprana }\end{array}$} \\
$\begin{array}{c}\text { Total de } \\
\text { historias } \\
\text { clínicas }\end{array}$ & Porcentaje & $\begin{array}{c}\text { Total de } \\
\text { historias } \\
\text { clínicas }\end{array}$ & Porcentaje \\
37 & $41,5 \%$ & 52 & $58,4 \%$ \\
\hline
\end{tabular}

En la Tabla 3 se presentan los 7 signos y síntomas que se tuvieron en cuenta en la encuesta para la clasificación de los pacientes con neumonía muy grave en niños menores de 2 meses, de los cuales se presentaron la saturación de oxígeno menor del $92 \%$ en $50 \%$ de los casos, e incapacidad para beber líquidos en $50 \%$.

En la Tabla 4 se presentan los 8 signos y síntomas tenidos en cuenta en la encuesta para la clasificación de los pacientes con neumonía muy grave en niños mayores de 2 meses. Los que se presentaron con mayor frecuencia fueron: retracciones torácicas en 33,33\%, seguido de convulsiones en $21,25 \% \mathrm{y}$, en una menor frecuencia, se encontró la desnutrición grave en $0,96 \%$ de los pacientes.

La razón de prevalencia calculada para el grupo de pacientes con ablactación temprana en niños menores de 2 meses fue de 1; es decir, que en nuestro estudio no se encontró relación entre este factor de riesgo con 
Tabla 3. Signos y síntomas de neumonía muy grave en pacientes menores de 2 meses

\begin{tabular}{|lcc|}
\hline \multicolumn{1}{|c}{ Signo/síntoma } & $\begin{array}{c}\text { Frecuencia de } \\
\text { presentación }\end{array}$ & Porcentaje \\
\hline $\begin{array}{l}\text { Saturación de oxígeno } \\
\text { menor del 92\% }\end{array}$ & 2 & $50 \%$ \\
$\begin{array}{l}\text { Incapacidad para beber } \\
\text { líquidos }\end{array}$ & 2 & $50 \%$ \\
\hline $\begin{array}{l}\text { Somnolencia importante } \\
\text { con dificultad para } \\
\text { despertar al niño }\end{array}$ & - & $0 \%$ \\
\hline Fiebre & - & $0 \%$ \\
\hline Hipotermia & - & $0 \%$ \\
Vomita todo & - & $0 \%$ \\
\hline Taquipnea $>60$ rpm & - & $0 \%$ \\
\hline $\begin{array}{l}\text { Disminución importante } \\
\text { del apetito }\end{array}$ & - & $0 \%$ \\
\hline Presencia de convulsión & - & $0 \%$ \\
\hline
\end{tabular}

la presentación de neumonía muy grave. Sin embargo, para el grupo de pacientes mayores de 2 meses hasta los 5 años, la razón de prevalencia fue de 1,4; lo que significa que los pacientes que tienen ablactación temprana poseen un riesgo de $40 \%$ de presentar neumonía muy grave con respecto a los pacientes $\sin$ ablactación temprana.

\section{Discusión}

Según los resultados obtenidos en el estudio se puede deducir que existe una relación importante entre la ablactación temprana y la presentación de neumonía muy grave en niños; en especial, en los mayores de 2 meses hasta los 5 años. Esto demuestra que la lactancia materna hasta la edad sugerida por la OMS protege al niño en las primeras etapas de vida frente a infecciones respiratorias. Cabe destacar que no se halló esta relación en los menores de 2 meses, probablemente la razón que explicaría este hecho es la no obtención de una muestra epidemiológicamente significativa en este grupo etario $(2,3)$.
Tabla 4. Signos y síntomas de neumonía muy grave en pacientes mayores de 2 meses hasta 5 años

\begin{tabular}{|lcc|}
\hline \multicolumn{1}{|c}{ Signo/síntoma } & $\begin{array}{c}\text { Frecuencia de } \\
\text { presentación }\end{array}$ & Porcentaje \\
\hline $\begin{array}{l}\text { Saturación de oxígeno } \\
\text { menor del 92\% }\end{array}$ & 15 & $7,24 \%$ \\
Cianosis & 12 & $5,79 \%$ \\
\hline $\begin{array}{l}\text { Somnolencia importante } \\
\text { con dificultad para } \\
\text { despertar al niño }\end{array}$ & 26 & $12,56 \%$ \\
$\begin{array}{l}\text { Incapacidad para beber } \\
\text { líquidos }\end{array}$ & 9 & $4,34 \%$ \\
\hline $\begin{array}{l}\text { Vomita todo } \\
\text { Desnutrición grave }\end{array}$ & 30 & $14,49 \%$ \\
\hline Presencia de convulsión & 44 & $0,96 \%$ \\
\hline $\begin{array}{l}\text { Presencia de retracciones } \\
\text { torácicas profundas }\end{array}$ & 69 & $21,25 \%$ \\
\hline
\end{tabular}

Según un estudio realizado por la Facultad de Salud Pública, Escuela de Medicina de la Escuela Superior Politécnica de Chimborazo, Ecuador, se determinó la ablactación temprana como un factor de riesgo para desarrollar neumonía, principalmente en niños menores de 5 años, debido a que la leche materna protege contra infecciones respiratorias a través de ciertos mecanismos, incluidos sustancias antivirales, antibacterianas, células inmunológicamente activas y los estimulantes del sistema inmunológico de los infantes. Según los resultados arrojados no sucede lo mismo con los niños que recibieron leche artificial sola o en conjunto con la leche materna, que representan el 13,5\%, y aquellos sometidos al destete prematuro antes de los 6 meses, que alcanzan el 14,2\% (4-6).

Dicho estudio respalda los resultados obtenidos en nuestro proyecto de investigación, el cual tiene como esencia revalorar la importancia de la lactancia materna exclusiva al menos los 6 primeros meses de vida del niño, siendo ideal, insustituible, suficiente, oportuna y adecuada (7-9). Finalmente es posible demostrar que 
la lactancia materna exclusiva durante los primeros 6 meses es recomendable, aceptada y avalada por la Organización Mundial de la Salud como factor protector importante para la prevención de infecciones respiratorias grave en los niños $(10,11)$.

\section{Conclusiones}

A partir de los resultados obtenidos en el estudio se puede concluir que existe una relación entre la ablactación temprana y la presentación de neumonía muy grave en los pacientes mayores de 2 meses hasta los 5 años, encontrando un $40 \%$ más de riesgo de presentar neumonía muy grave en estos pacientes, con respecto a aquellos sin ablactación temprana. En los pacientes menores de 2 meses no se encontró la relación entre ablactación temprana y la presentación de neumonía muy grave. Se concluyó que el signo de neumonía muy grave que con mayor frecuencia se presentó fue el de las retracciones torácicas profundas en $33,3 \%$, seguido de convulsiones en $21,2 \%$.

Con este estudio se apoya la recomendación de la lactancia materna exclusiva hasta los 6 meses de edad formulada por la OMS, ya que es un factor protector importante para la prevención de infecciones respiratorias graves y para la protección del niño frente a enfermedades infecciosas respiratorias graves, mediante el aporte de inmunoglobulinas y anticuerpos que aumentan la respuesta inmunitaria.

\section{Agradecimientos}

A los siguientes colaboradores que hicieron posible dicha investigación: Danilo Cambronell C, Mayra Castilla M, Yorleidis Díaz G, Arniel Hernández M, Lili Hernández S, José Martínez G, Liceth Mercado S, Mónica Quintero G, Karen Tejada A, Luis Torres V, José Villalba G.

\section{Conflicto de interés}

Los autores declaran no tener ningún conflicto de interés.

\section{Referencias}

1. Bradley JS, Byington CL, Shah SS, Alverson B, Carter ER, Harrison C, et al. The Management of Community-Acquired Pneumonia in Infants and Children Older Than 3 Months of Age: Clinical Practice Guidelines by the Pediatric Infectious Diseases Society and the Infectious Diseases Society of America. Clin Infect Dis. 2011;e1-52. Doi: 10.1093/cid/cir531.

2. McIntosh K. Community-acquired pneumonia in children. N Engl J Med. 2002;346:429-437. DOI: 10.1056/NEJMra011994.

3. Asociación Colombiana de Neumología Pediátrica. Tratamiento de la neumonía adquirida en la comunidad de niños de 1 mes a 17 años. 2010.

4. Wardlaw TM, Johansson EW, Hodge MJ. UNICEF, Division of Communication and World Health Organization. Pneumonia: the forgotten killer of children. New York: UNICEF. Geneva World Health Organization. 2006.

5. Isturiz R, Luna C, Ramírez J. Clinical and economic burden of pneumonia among adults in Latin America. Int J InfectDis. 2010;14(10):e852-6. DOI: 10.1016/j.ijid.2010.02.2262.

6. Martín AA, Moreno-Pérez D, Miguélez SA, Gianzo JA, García ML, Murua JK, et al. Etiología y diagnóstico de la neumonía adquirida en la comunidad y sus formas complicadas. An Pediatr (Barc). 2012;76(3):162e1-18. DOI: 10.1016/j.anpedi.2011.09.011.

7. Agudelo A, Manotas M, Vásquez C. Neumonía adquirida en la comunidad en niños. Precop SCP. 2010;10(3):16-27.

8. Úbeda Sansano MI, Murcia García J, Asensi Monzó MT. Neumonía adquirida en la comunidad. Protocolos del GVR (publicación P-GVR-8). Disponible en: https://www.aepap.org/ sites/default/files/gvr/protocolo-neumonia-2011.pdf

9. Organización Mundial de la Salud; Organización Panamericana de la Salud. Atención Integrada a las Enfermedades Prevalentes de la Infancia. 2.a edición. Bogotá, Colombia: Organización Panamericana de la Salud; 2015.

10. McInerny T, Adam H, Campbell D, Kamat D, Kelleher K, Hoekelman R. Tratado de pediatría. New York: Médica Panamericana. 2011.

11. Ministerio de Protección Social. Plan decenal lactancia materna 2010-2020. Disponible en: https://www.minsalud.gov. $\mathrm{co} /$ sites/rid/Lists/BibliotecaDigital/RIDE/VS/PP/SNA/plandecenal-lactancia-materna.pdf 